\title{
The effect of citrate dialysate on intradialytic heparin dose in haemodialysis patients: study design of a randomised controlled trial
}

\author{
Davina J. Tai ${ }^{1}$, Kelvin Leung ${ }^{2}$, Pietro Ravani ${ }^{2,3}$, Robert R. Quinn ${ }^{2,3}$, Nairne Scott-Douglas ${ }^{2}$, Jennifer M. MacRae ${ }^{2 *}$ and \\ For the Alberta Kidney Disease Network
}

\begin{abstract}
Background: Unfractionated heparin is the most common anticoagulant used in haemodialysis (HD), although it has many potential adverse effects. Citrate dialysate (CD) has an anticoagulant effect which may allow reduction in cumulative heparin dose (CHD) compared to standard acetate dialysate (AD).

Methods: This double-blinded, randomised, cross-over trial of chronic haemodialysis patients determines if CD allows reduction in CHD during HD compared with AD. After enrolment, intradialytic heparin is minimised during a two-week run-in period using a standardised protocol based on a visual clotting score. Patients still requiring intradialytic heparin after the run-in period are randomised to two weeks of HD with AD followed by two weeks of $C D$ (Sequence 1) or two weeks of HD with CD followed by two weeks of AD (Sequence 2). The primary outcome is the change in CHD with CD compared with AD. Secondary outcomes include metabolic and haemodynamic parameters, and dialysis adequacy.
\end{abstract}

Discussion: This randomised controlled trial will determine the impact of CD compared with AD on CHD during HD.

Trial registration: ClinicalTrials.gov NCT01466959

Keywords: Citrate dialysate, Hemodialysis, Haemodialysis, Unfractionated heparin, Anticoagulation, Randomised controlled trial

\section{Background}

During haemodialysis (HD), blood is exposed to an extracorporeal circuit which activates thrombogenic pathways and clotting [1]. Clotting in the dialysis circuit decreases HD efficiency, and increases nursing workload and costs [2]. Even subclinical clotting of the dialysis circuit may reduce effective dialyser surface area and pore size, decreasing both small and middle solute clearance. Additionally, activation of the coagulation pathway in HD patients may be associated with increased inflammation and accelerated atherosclerosis [3].

In North America, unfractionated heparin is the most common anticoagulant used to prevent clotting during

\footnotetext{
* Correspondence: jennifer.macrae@albertahealthservices.ca

${ }^{2}$ Department of Medicine, University of Calgary, Calgary, Alberta, Canada

Full list of author information is available at the end of the article
}

HD $[4,5]$. Although effective and inexpensive, it has a narrow therapeutic window without adverse bleeding. Since heparin provides systemic anticoagulation, it is contraindicated in the setting of active bleeding, trauma, pericarditis, intracerebral haemorrhage, thrombocytopenia, coagulopathy, and peri-operative care [6-9]. Furthermore, heparin may lead to osteoporosis, dyslipidaemia, hyperkalaemia, and immune-mediated heparin-induced thrombocytopenia $[2,10-16]$.

An alternative to systemic heparin anticoagulation for HD is citrate dialysate (CD). CD contains a small amount of citric acid rather than acetic acid as the acidifying agent, at one-fifth the concentration of citric acid used for regional citrate anticoagulation. There are several observational studies [17-27] and two randomised trials using $\mathrm{CD}$ in $\mathrm{HD}$ patients $[28,29]$. These studies demonstrate that $\mathrm{CD}$ is associated with decreased clotting of the haemodialysis circuit [17], decreased need for
C Biomed Central

(C) 2015 Tai et al. Open Access This article is distributed under the terms of the Creative Commons Attribution 4.0 International License (http://creativecommons.org/licenses/by/4.0/), which permits unrestricted use, distribution, and reproduction in any medium, provided you give appropriate credit to the original author(s) and the source, provide a link to the Creative Commons license, and indicate if changes were made. The Creative Commons Public Domain Dedication waiver (http://creativecommons.org/publicdomain/zero/1.0/) applies to the data made available in this article, unless otherwise stated. 
anticoagulation [24, 26, 29], increased dialyser reuse [19], and increased small and middle solute clearance [18-20, $26-28,30]$. These benefits are from an anticoagulation effect that is limited to the extracorporeal circuit without systemic coagulation activation [28]. Other associated benefits of CD include decreased acidosis [18, 22, 28], decreased inflammation [27], decreased oxidative stress [25], improved anaemia [22], improved nutrition [22], and greater haemodynamic stability [21, 28, 31]. CD is well tolerated with no unstable metabolic parameters and no documented adverse effects $[18,22,29]$, even in paediatric patients with acute kidney injury [32], in critically ill patients [17], and patients with advanced liver failure [33].

We describe our protocol for a six-week, doubleblinded, randomised cross-over trial that investigates whether $\mathrm{CD}$ results in reduced cumulative heparin dose (CHD) compared to acetate dialysate (AD) in chronic HD patients. Secondary outcomes address safety concerns, and include metabolic and haemodynamic parameters, and dialysis adequacy.

\section{Methods \\ Study setting}

This randomised crossover clinical trial is performed within three community HD units in Calgary, Alberta, Canada, where unfractionated heparin is used for standard intradialytic anticoagulation. All participants provide written informed consent. The study protocol is approved by the Conjoint Health Research Ethics Board at the University of Calgary, and is conducted in accordance with the Declaration of Helsinki (ClinicalTrials.gov, NCT01466959).

\section{Study population}

Eligible patients are $\geq 18$ years, and on outpatient conventional $H D$ three times per week for at least three months. Exclusion criteria include: contraindication to heparin, heparin-free HD, known clotting disorder, warfarin therapy, dialysing with a dysfunctional central venous catheter (blood flow rate consistently $<300 \mathrm{~mL} / \mathrm{min}$ and/or frequent use of thrombolytic), vascular access dysfunction, planned vascular access conversion or procedure during the study period, use of high calcium dialysate, active medical issue, planned kidney transplant during the study period, planned conversion of dialysis modality (peritoneal dialysis, nocturnal dialysis) during the study period, or inability to provide informed consent. Patients with recent modifications to their erythropoiesis-stimulating agent dose are reviewed by the investigator for eligibility.

\section{Study protocol}

After enrolment, each patient's dose of intradialytic heparin is minimised during a two-week run-in period
(Fig. 1) using study protocol (Table 1) which is based on a standardised visual clotting score [34]. The visual clotting score is quantified by inspecting the venous chamber and all four quadrants of the dialyser at the end of the HD session (0: clear dialyser/no clots; 1 : few strands/ small clots; 2: half clotted; 3: three-quarters clotted; 4: completely clotted, unable to return blood) (Table 1 ). The heparin dose is reduced to reach a target visual clotting score of 2, then increased slightly and maintained, to achieve the lowest possible dose required to prevent clotting (Table 1). The CHD during the last HD session of the run-in period is considered to be the patient's baseline heparin dose. Patients still requiring intradialytic heparin after the two-week run-in period, and who still meet inclusion and exclusion criteria, are randomised to one of two treatment sequences (Fig. 1). For sequence 1, patients undergo six HD sessions over two weeks with $\mathrm{AD}$ (control), followed by six HD sessions over two weeks with CD (intervention). For sequence 2, patients undergo six HD sessions over two weeks with $\mathrm{CD}$ (intervention), followed by two weeks of $\mathrm{AD}$ (control) (Fig. 1). Patients begin each study phase (AD and $\mathrm{CD})$ with their baseline intradialytic heparin dose, determined at the end of the run-in period. Nurses subsequently adjust intradialytic heparin dose per protocol (Table 1) during each HD session of each study phase. At the end of the first study phase (two weeks), a 68-h washout period ensures no potential for a carry-over effect. After the wash-out period, patients cross-over to the other treatment for the next two weeks beginning again with their baseline heparin dose (Fig. 1). A twoweek duration for each strategy is chosen to ensure adequate time to attain the minimum required heparin dose.

Randomisation is determined by a computer generated random number list and concealed in sequentially numbered, opaque, sealed envelopes to ensure allocation concealment. Following informed consent and enrolment, the envelope containing allocation sequence is provided to the research coordinator who implements the sequence assignment according to protocol. The intervention and control dialysates, $\mathrm{CD}$ and $\mathrm{AD}$, are packaged identically by the manufacturer (Chief Medical Supplies, Ltd., Calgary, Alberta, Canada) for blinding of investigators, patients, and health care staff. All data is confidential, and entered into electronic records by an independent data entry clerk who is blinded to patient allocation.

All study patients are dialysed using Gambro Phoenix ${ }^{\circ}$ dialysis systems (Gambro-Hospal, Mirandola, Italy) at least three times per week. Each patient's target weight and dialysis prescription are optimised at the study start by the rounding nephrologist using clinical assessment. Standard dialysis prescriptions (blood flow rate $300-$ $450 \mathrm{~mL} / \mathrm{min}$, dialysate flow rate $500 \mathrm{~mL} / \mathrm{min}$, dialysate temperature $0.5{ }^{\circ} \mathrm{C}$ less than patient temperature, 


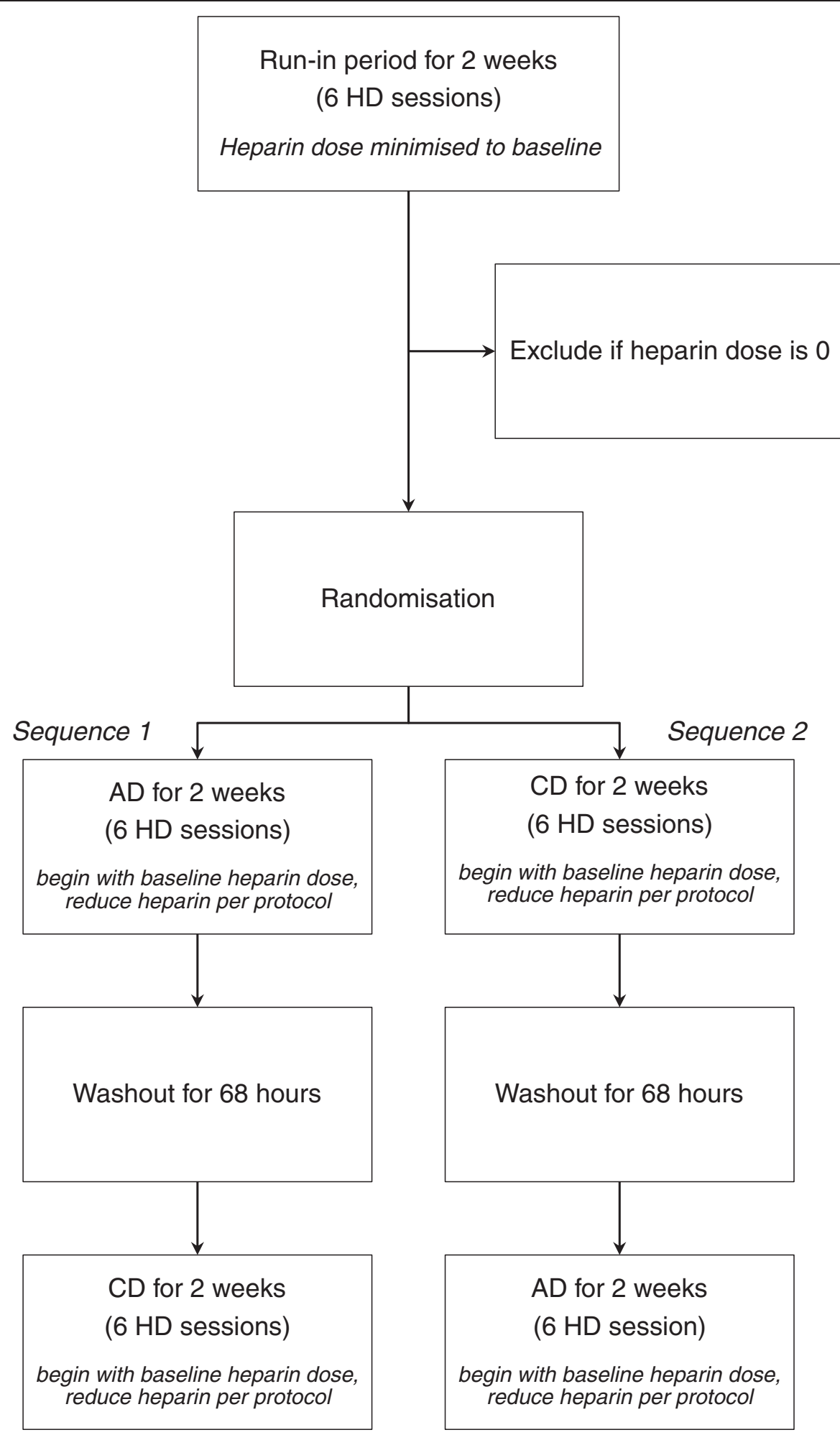

Fig. 1 Study Flow Diagram

dialysate sodium $137 \mathrm{mmol} / \mathrm{L}$ ) are maintained unless otherwise prescribed. Dialysers are high-flux, polysulfone (REXEED $^{\mathrm{mm}}$, Asahi Kasei Medical Co. Ltd., Tokyo, Japan).
HD prescriptions, target weights, and antihypertensive medications are not changed during the study period. The intervention dialysate, $\mathrm{CD}$, contains $2.4 \mathrm{mEq} / \mathrm{L}$ of 
Table 1 Visual Clotting Score and Heparin Protocol

\begin{tabular}{|c|c|c|}
\hline Clotting Score & $\begin{array}{l}\text { DialyserNenous Chamber Appearance } \\
\text { (at the end of each HD session) }\end{array}$ & Heparin Dose Adjustment (for the next HD session) \\
\hline 0 & Clear & Decrease ${ }^{a}$ boost by 200 units and decrease running dose by 200 units $/ \mathrm{h}$ \\
\hline 1 & Few strands/small clot & Decrease ${ }^{a}$ boost by 200 units and decrease running dose by 200 units $/ h$ \\
\hline 2 & $1 / 2$ clotted & $\begin{array}{l}\text { Increase boost by } 200 \text { units and increase running dose by } 200 \text { units/h and } \\
\text { maintain this new heparin dose for the remainder of this study phase only. }\end{array}$ \\
\hline 3 & $3 / 4$ clotted (able to return blood) & Increase boost by 300 units and increase running dose by 300 units/h \\
\hline 4 & Completely clotted (unable to return blood) & Increase boost by 400 units and increase running dose by 400 units/h \\
\hline \multicolumn{3}{|c|}{ 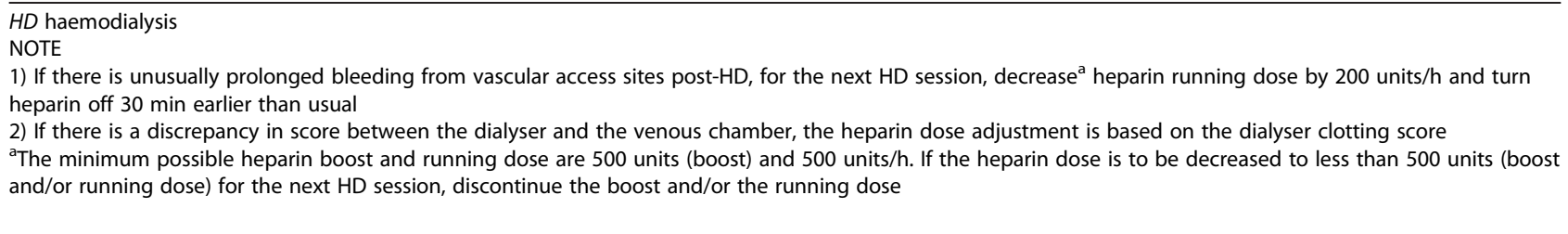 } \\
\hline
\end{tabular}

citric acid (Citrasate ${ }^{\bullet}$ Advanced Renal Technologies, Bellevue, WA), and the control dialysate, AD, contains $4 \mathrm{mmol} / \mathrm{L}$ of acetic acid.

\section{Study endpoints}

Primary outcome measures are the absolute and percent change in CHD from baseline with $\mathrm{CD}$ compared with $\mathrm{AD}$. CHD is defined as the total heparin received per dialysis session. Secondary outcomes are the effect of CD compared with $\mathrm{AD}$ on systemic anticoagulation (prothrombin time $[\mathrm{PT}] /$ international normalised ratio [INR], activated partial thromboplastin time [aPTT]), intradialytic metabolic parameters (serum total serum calcium, ionized calcium, magnesium, bicarbonate), anaemia, haemodynamic stability (blood pressure, heart rate, corrected QT interval, number of HD sessions complicated by intradialytic hypotension), dialysis adequacy (single pool Kt/V, urea reduction ratio, beta-2-microglobulin [B2M]), systematic inflammation (C-reactive protein [CRP]), bleeding time after $\mathrm{HD}$, and bleeding events. Also, the visual clotting score and the dialyser fibre bundle volume (FBV) (as measured by the ultrasound dilution technique [35]) are correlated. Lastly, the intra-rater and inter-rater reliability of the visual clotting score is assessed.

\section{Data collection}

\section{Baseline characteristics}

Baseline patient demographics (age, gender, HD vintage, race), comorbidities, cause of ESRD, labs, and HD prescription (dialyser, dialysate composition and temperature, blood and dialysate flow rates, target weight, anticoagulation) are extracted from HD charts and the Southern Alberta Renal Program database [36].

\section{Cumulative heparin dose}

The CHD is calculated per HD session, and determined from HD run sheets. Specifically, CHD is the initial heparin bolus, plus the hourly heparin infusion rate multiplied by the time on HD during which heparin is infused, per session, in heparin units. For the primary outcome, the CHD for the last two HD runs of each study phase are recorded, averaged, and used to calculate change from baseline CHD. The last two HD sessions of each phase is chosen to allow adequate time in each phase for heparin dose minimisation.

\section{Visual clotting score, inter-and intra-rater reliability}

The clotting score is a visual grade of dialyser and venous chamber clotting (Table 1). To ensure consistency, scoring of dialyser/venous chamber clotting for heparin dose adjustments are performed at the end of each HD session by a single, trained research nurse. A second HD nurse independently and blindly scores the dialyser/venous chamber appearance; this second clotting score is recorded to assess inter-rater reliability, but is not used to adjust the heparin dose per protocol. To assess for intra-rater reliability, at the end of each HD session during the run-in phase, the dialyser quadrants are captured in four photographs by the same trained research nurse, using a digital camera on a tripod, with standardised lighting, at a standardised distance and height from the dialyser. The dialyser is rotated by $90^{\circ}$ for each of the four photographs, to visually capture the full circumferential view of the dialyser. The photographs are represented in a blinded fashion to the same research nurse at a later date to be re-scored.

\section{Metabolic, anticoagulation, and inflammatory parameters} Serum total calcium, ionized calcium, albumin, magnesium, bicarbonate, haemoglobin, urea, PT/INR, aPTT, $\mathrm{B} 2 \mathrm{M}$, and CRP are collected at the beginning of the first HD session of the study (whether randomised to Sequence 1 or 2), and drawn again both pre and post HD for the last HD sessions of each study phase. Ionized 
calcium is drawn pre and post HD during the first and last HD session in each study phase.

\section{Haemodynamic parameters}

Blood pressure and heart rate are measured and recorded by HD nurses pre and post HD, and every 30 min during HD. The number of HD sessions complicated by intradialytic hypotension (IDH) during each study phase is recorded. IDH is defined as a drop in the systolic blood pressure (SBP) by $\geq 20 \mathrm{mmHg}$ to $<$ $100 \mathrm{mmHg}$ with patient symptoms or nursing/physician intervention. If the patient's pre-HD SBP is $<100 \mathrm{mmHg}$, then IDH is defined as a drop in $\mathrm{SBP} \geq 10 \mathrm{mmHg}$ with patient symptoms or nursing/physician intervention. An electrocardiogram is performed at baseline (after the run-in phase), as well as pre and post HD during the last HD session in each two week study phase.

\section{Dialysis adequacy}

Single pool Kt/V is determined for each HD session using Diascan $^{\circ}$ (Hospal-Gambro, Mirandola, Italy), which measures the dialysance of sodium through the dialyser membrane corrected for recirculation and ultrafiltration [37], and which is a validated method for measuring urea clearance [38]. In addition, the urea reduction ratio is calculated for the last HD session of each study phase using the equation ((urea pre-urea post)/urea pre).

\section{Bleeding time and bleeding events}

For the last two HD sessions of each study phase, the bleeding time post-HD is recorded and averaged in patients who have a fistula or graft as their vascular access. Bleeding events are documented and classified into: 1) major bleeding (fatal bleeding, overt bleeding associated with a haemoglobin drop $\geq 20 \mathrm{~g} / \mathrm{L}$ and/or transfusion with 2 units of red blood cells, retroperitoneal/intracranial/intraspinal/intraocular/pericardial/non-traumatic intra-articular bleeding), 2) clinically important non-major bleeding (clinically overt bleeding requiring hospital admission or visit to a medical facility or leading to intervention), and 3) minor bleeding (any bleeding episode not meeting criteria for the preceding bleeding categories).

\section{Fibre bundle volume}

FBV is the space within the blood compartment of hollow fibre dialysers [35]. Using the ultrasound dilution technique (Transonic ${ }^{\circ}$ HD02 monitor, Transonic Systems Inc. ${ }^{\oplus}$, Ithaca, NY, USA), dialyser FBV is measured twice (and the mean recorded) during both the first and last $30 \mathrm{~min}$ of the mid-week HD sessions throughout the entire study period (including the run-in phase). The change in FBV during HD represents the quantity of clot in the dialyser [35] and is determined using the equation ((FBV pre-FBV post)/FBV pre).

\section{Sample size calculations}

Previous studies have demonstrated that CHD is reduced by $30 \%$ with the use of $\mathrm{CD}$ [39]. Locally in Calgary, the mean CHD is 3000 units (standard deviation 900 units) per HD session in $85 \%$ of HD patients. Based on this, and taking into account a $30 \%$ dropout rate, we estimate that 20 patients are required to demonstrate a $30 \%$ (1000 units) reduction in $\mathrm{CHD}$ with $\mathrm{CD}$ compared to $\mathrm{AD}$ (alpha 0.05 , power $80 \%$ ). We use the method of Frison and Pocock for the ANCOVA model, and Rabe-Hesketh and Skrondal for the mixed linear model solution (StataCorp LP version SE 11.0, College Station, TX, USA).

\section{Statistical analysis}

Baseline characteristics will be presented as means and $95 \%$ confidence intervals (CIs) or medians and interquartile range (IQR) for continuous variables, and absolute and relative proportions for categorical variables. To determine the effect of $\mathrm{CD}$ compared with $\mathrm{AD}$ on reduction in CHD (the primary outcome) a random intercept linear mixed model will be used, with $\mathrm{CD}$ or $\mathrm{AD}$ as the main exposure, subjects as source of random effects, baseline heparin dose values as covariate (at least 2 of 3 measures from the first of two weeks of each period), and final heparin dose values as outcome (at least 2 of 3 measures of the second week of each period). This model takes into account the correlation of data caused by repeated measures for each subject given the crossover design of the study. Treatment, treatment period, and treatment sequence will be modelled as fixed effects. If there is no evidence of period or carry-over effects, all comparisons will be presented relative to $\mathrm{AD}$, without the need for consideration of carry-over effects.

\section{Discussion}

CD has the potential to decrease intradialytic anticoagulation requirements, and improve patient outcomes. However, existing literature comparing $\mathrm{CD}$ to $\mathrm{AD}$ is limited. Most studies are small, observational in nature, and focus on dialysis adequacy, dialyser reuse/clotting, or metabolic/inflammatory/haemodynamic parameters $[17-23,25,27]$. Only two prospective randomised studies comparing $\mathrm{CD}$ to $\mathrm{AD}$ have been performed. One single-centre, unblinded crossover study randomised twenty-three maintenance HD patients to first receive $\mathrm{CD}$ without anticoagulation or $\mathrm{AD}$ with a Tinzaparin bolus [29]. If the $\mathrm{CD}$ session revealed early clotting of the venous chamber or dialyser, a Tinzaparin bolus at half the usual dose could be given, and repeated once. During the HD sessions with CD, $50 \%$ of patients did not require anticoagulation, $32 \%$ required a reduced Tinzaparin bolus, and $18 \%$ stopped HD early due to clotting without Tinzaparin. Of those who received anticoagulation with $\mathrm{CD}$, the median dose of Tinzaparin 
received was $40 \%$ of the standard dose. There was no difference in dialysis adequacy between groups [29]. The main limitation of this study is that the two groups were not treated equally; unlike the $\mathrm{CD}$ group, no attempt was made to reduce anticoagulation in the AD group [29]. Another single-centre, single-blinded, crossover study randomised twenty-five clinically stable maintenance HD patients to receive either $\mathrm{AD}$ or $\mathrm{CD}$ weekly in an alternating fashion for four weeks [28]. The primary outcome was systemic haemodynamics; secondary outcomes were coagulation activation, acid-base balance, calcium balance, and dialysis adequacy. Compared with $\mathrm{AD}$, the $\mathrm{CD}$ group had significantly lower blood pressure and peripheral vascular resistance without any difference in stroke volume. However, the decrease in SBP was ablated with dialysate calcium supplementation in the last study week. $\mathrm{CD}$ resulted in a significant increase in pre-HD bicarbonate levels, lower post-HD ionized calcium, and higher $\mathrm{Kt} / \mathrm{V}$ without any effect on coagulation parameters [28]. This study did not assess the effect of $\mathrm{CD}$ on intradialytic heparin dose (heparin was used per usual protocol in both groups); the effect of CD on IDH was also not examined [28].

Four observational studies specifically address heparin use with $C D$ in maintenance HD patients [24, 26, 30,39]. One study included 277 chronic HD patients from eight HD units in an eight-week long, open-label, sequential four-phase study [26]. The primary outcome was change in mean effective conductivity clearance with $C D$ (and reduced heparin) versus $\mathrm{AD}$ (and standard heparin dose). Each study phase was two weeks long (6 HD sessions). In the first baseline phase, patients received $\mathrm{AD}$ and $100 \%$ of their standard heparin dose. In subsequent phases, patients received $\mathrm{CD}$ and decreasing amounts of heparin (second phase: $\mathrm{CD}$ and $100 \%$ of standard heparin dose; third phase: $\mathrm{CD}$ and $80 \%$ of standard heparin dose; fourth phase: $C D$ and $2 / 3$ of standard heparin dose). At the end of the study, there was no difference in dialysis adequacy between baseline (AD with $100 \%$ heparin) and subsequent phases (CD with decreased heparin). Even with a $33 \%$ reduction in heparin, there was no difference in HD circuit clotting, and dialysis adequacy was maintained [26]. This study did not assess if a reduced heparin dose was possible with $\mathrm{AD}$ [26]. In another prospective study, chlorhydric-acid based dialysate was compared with $\mathrm{CD}$ in ten patients treated with post-dilution online haemodiafiltration [24]. During treatments with CD, the heparin dose was decreased by half, then completely discontinued. In $120 \mathrm{CD}$ sessions without heparin, dialysis adequacy was unchanged, and only one clotting episode related to vascular access stenosis was experienced [24]. Two further observational studies, published in abstract form only, included 20 and 31 chronic HD patients, respectively, who had prolonged bleeding from vascular access needling sites post HD. Patients were switched from $\mathrm{AD}$ to $\mathrm{CD}$, and intradialytic heparin doses were reduced by $30 \%[19,39]$ after two months, and $55 \%$ [30] after four months, respectively. In both studies, heparin reduction with $\mathrm{CD}$ was achieved without decrease in dialysis adequacy [30, 39].

Although existing literature suggests that $\mathrm{CD}$ use during chronic HD allows intradialytic heparin reduction without sacrifice of dialysis dose, and without other adverse effects, this has yet to be investigated in a rigorous trial. We have presented in detail the motivation, design and strategy for our randomised controlled trial, including methods of recruitment, randomisation, allocation concealment, dialysis intervention, outcome assessment, and data collection. The study protocol is developed according to Standard Protocol Items: Recommendations for Interventional Trials (SPIRIT) 2013 [40]; results will be reported following the Consolidated Standards of Reporting Trials (CONSORT) statement [41].

In summary, our randomised crossover trial of chronic HD patients will determine if $\mathrm{CD}$ decreases intradialytic heparin requirements, and its affect on haemodynamic stability and dialysis adequacy. Our study will answer a clinically relevant and important question which has not previously been addressed by a well-designed randomised trial.

\section{Abbreviations}

HD: Haemodialysis; CD: Citrate dialysate; CHD: Cumulative heparin dose; AD: Acetate dialysate; PT: Prothrombin time; INR: International normalised ratio; aPTT: Activated partial thromboplastin time; B2M: Beta-2-microglobulin; CRP: C-reactive protein; FBV: Fibre bundle volume; IDH: Intradialytic hypotension; SBP: Systolic blood pressure; Cls: Confidence intervals; IQR: Interquartile range.

\section{Competing interests}

The authors declare that they have no competing interests.

\section{Authors' contributions}

DT participated in the study design, and drafted the protocol manuscript. KL participated in data acquisition, and will participate in data analysis. PR participated in the study design and will perform statistical analysis. RQ participated in the study design. NSD participated in the study design and coordination. JM conceived of the study and participated in its design and coordination. All authors read and approved the final protocol manuscript.

\section{Acknowledgements}

This study was funded by the Division of Nephrology, University of Calgary.

\section{Disclosures}

The authors have no financial interests in the information contained in this manuscript.

\section{Author details}

'Department of Medicine, University of Saskatchewan, Saskatoon, Saskatchewan, Canada. ${ }^{2}$ Department of Medicine, University of Calgary, Calgary, Alberta, Canada. ${ }^{3}$ Department of Community Health Sciences, University of Calgary, Calgary, Alberta, Canada.

Received: 20 November 2014 Accepted: 13 August 2015

Published online: 25 August 2015 


\section{References}

1. Fischer KG. Essentials of anticoagulation in hemodialysis. Hemodial Int. 2007;11(2):178-89.

2. Shen Jl, Winkelmayer WC. Use and safety of unfractionated heparin for anticoagulation during maintenance hemodialysis. Am J Kidney Dis. 2012;60(3):473-86

3. Pawlak K, Naumnik B, Brzosko S, Pawlak D, Mysliwiec M. Oxidative stress - a link between endothelial injury, coagulation activation, and atherosclerosis in haemodialysis patients. Am J Nephrol. 2004;24(1):154-61.

4. Cronin RE, Reilly RF. Unfractionated heparin for hemodialysis: still the best option. Semin Dial. 2010;23(5):510-5.

5. Brunet $P$, Simon N, Opris A, Faure V, Lorec-Penet AM, Portugal $H$, et al. Pharmacodynamics of unfractionated heparin during and after a hemodialysis session. Am J Kidney Dis. 2008;51(5):789-95.

6. Wasse H, Gillen DL, Ball AM, Kestenbaum BR, Seliger SL, Sherrard D, et al. Risk factors for upper gastrointestinal bleeding among end-stage renal disease patients. Kidney Int. 2003;64(4):1455-61.

7. Chachati A, Godon JP. Effect of haemodialysis on upper gastrointestinal tract pathology in patients with chronic renal failure. Nephrol Dial Transplant. 1987;1(4):233-7.

8. Yang JY, Lee TC, Montez-Rath ME, Paik J, Chertow GM, Desai M, et al. Trends in acute nonvariceal upper gastrointestinal bleeding in dialysis patients. $J$ Am Soc Nephrol. 2012;23(3):495-506.

9. Seliger SL, Gillen DL, Longstreth Jr WT, Kestenbaum B, Stehman-Breen CO. Elevated risk of stroke among patients with end-stage renal disease. Kidney Int. 2003;64(2):603-9.

10. Davenport A. Antibodies to heparin-platelet factor 4 complex: pathogenesis, epidemiology, and management of heparin-induced thrombocytopenia in hemodialysis. Am J Kidney Dis. 2009;54(2):361-74.

11. Nasstrom B, Olivecrona G, Olivecrona T, Stegmayr BG. Lipoprotein lipase during heparin infusion: lower activity in hemodialysis patients. Scand J Clin Lab Invest. 2003:63(1):45-53.

12. Schulman S, Hellgren-Wangdahl M. Pregnancy, heparin and osteoporosis. Thromb Haemost. 2002;87(2):180-1.

13. Binici DN, Gunes N. Risk factors leading to reduced bone mineral density in hemodialysis patients with metabolic syndrome. Ren Fail. 2010;32(4):469-74.

14. Lai KN, Ho K, Cheung RC, Lit LC, Lee SK, Fung KS, et al. Effect of low molecular weight heparin on bone metabolism and hyperlipidemia in patients on maintenance hemodialysis. Int J Artif Organs. 2001;24(7):447-55.

15. Edes TE. Heparin-induced hyperkalemia. Postgrad Med. 1990;87(4):104-6.

16. Hottelart C, Achard JM, Moriniere P, Zoghbi F, Dieval J, Fournier A. Heparininduced hyperkalemia in chronic hemodialysis patients: comparison of low molecular weight and unfractionated heparin. Artif Organs. 1998;22(7):614-7.

17. Tu A, Ahmad S. Heparin-free hemodialysis with citrate-containing dialysate in intensive care patients. Dial Transplant. 2000;29:620-6.

18. Ahmad S, Callan R, Cole JJ, Blagg CR. Dialysate made from dry chemicals using citric acid increases dialysis dose. Am J Kidney Dis. 2000;35(3):493-9.

19. Ahmad S, Callan R, Cole J, Blagg C. Increased dialyzer reuse with citrate dialysate. Hemodial Int. 2005;9(3):264-7.

20. Kossmann RJ, Gonzales A, Callan R, Ahmad S. Increased efficiency of hemodialysis with citrate dialysate: a prospective controlled study. Clin J Am Soc Nephrol. 2009;4(9):1459-64.

21. Daimon S, Dan K, Kawano M. Comparison of acetate-free citrate hemodialysis and bicarbonate hemodialysis regarding the effect of intra-dialysis hypotension and post-dialysis malaise. Ther Apher Dial. 2011;15(5):460-5.

22. Kuragano T, Kida A, Furuta M, Yahiro M, Kitamura R, Otaki Y, et al. Effects of acetate-free citrate-containing dialysate on metabolic acidosis, anemia, and malnutrition in hemodialysis patients. Artif Organs. 2012;36(3):282-90.

23. Kuragano T, Furuta M, Yahiro M, Kida A, Otaki Y, Hasuike $Y$, et al. Acetate free citrate-containing dialysate increase intact-PTH and BAP levels in the patients with low intact-PTH. BMC Nephrol. 2013;14:18-2369-14-18.

24. Aniort J, Petitclerc T, Creput C. Safe use of citric acid-based dialysate and heparin removal in postdilution online hemodiafiltration. Blood Purif. 2012:34(3-4):336-43.

25. Masuda A, Hagiwara S, Tanimoto M, Kodama F, Okumura K, Nohara N, et al. Effects of acetate-free citrate dialysate on glycoxidation and lipid peroxidation products in hemodialysis patients. Nephron Extra. 2012;2(1):256-68.

26. Sands JJ, Kotanko P, Segal JH, Ho CH, Usvat L, Young A, et al. Effects of citrate acid concentrate (citrasate $(\mathrm{R}))$ on heparin $\mathrm{N}$ requirements and hemodialysis adequacy: a multicenter, prospective noninferiority trial. Blood Purif. 2012;33(1-3):199-204
27. Panichi V, Fiaccadori E, Rosati A, Fanelli R, Bernabini G, Scatena A, et al. Postdilution on line haemodiafiltration with citrate dialysate: first clinical experience in chronic dialysis patients. ScientificWorld J. 2013;2013:703612.

28. Gabutti L, Lucchini B, Marone C, Alberio L, Burnier M. Citrate- vs, acetatebased dialysate in bicarbonate haemodialysis: consequences on haemodynamics, coagulation, acid-base status, and electrolytes. BMC Nephrol. 2009;10:7-2369-10-7.

29. Stegmayr BG, Jonsson P, Mahmood D. A significant proportion of patients treated with citrate containing dialysate need additional anticoagulation. Int J Artif Organs. 2013;36(1):1-6.

30. Kossman RJ, Callan R, Ahmad S. Fifty-five percent heparin reduction is sate with citrate dialysate in chronic dialysis patients [abstract]. American Society of Nephrology Annual Meeting 2006

31. Saito T, Saito O, Maeda T, Ito C, Ando Y, Yamagata T, et al. Metabolic and hemodynamic advantages of an acetate-free citrate dialysate in a uremic case of congenital methylmalonic acidemia. Am J Kidney Dis. 2009:54(4):764-9.

32. Hanevold C, Lu S, Yonekawa K. Utility of citrate dialysate in management of acute kidney injury in children. Hemodial Int. 2010;14 Suppl 1:S2-6.

33. Winrow RM, Davis C, Halldorson JB, Ahmad S. Intraoperative dialysis during liver transplantation with citrate dialysate. Hemodial Int. 2009:13(3):257-60.

34. Sagedal S, Hartmann A, Osnes K, Bjornsen S, Torremocha J, Fauchald P, et al. Intermittent saline flushes during haemodialysis do not alleviate coagulation and clot formation in stable patients receiving reduced doses of dalteparin. Nephrol Dial Transplant. 2006;21(2):444-9.

35. Krivitski NM, Kislukhin W, Snyder JW, MacGibbon DR, Kuznetsova OA, Reasons AM, et al. In vivo measurement of hemodialyzer fiber bundle volume: theory and validation. Kidney Int. 1998;54(5):1751-8.

36. Manns BJ, Mortis GP, Taub KJ, McLaughlin K, Donaldson C, Ghali WA. The Southern Alberta Renal Program database: a prototype for patient management and research initiatives. Clin Invest Med. 2001;24(4):164-70.

37. Petitclerc T, Goux N, Reynier AL, Bene B. A model for non-invasive estimation of in vivo dialyzer performances and patient's conductivity during hemodialysis. Int J Artif Organs. 1993;16(8):585-91.

38. Kuhlmann U, Goldau R, Samadi N, Graf T, Gross M, Orlandini G, et al. Accuracy and safety of online clearance monitoring based on conductivity variation. Nephrol Dial Transplant. 2001;16(5):1053-8.

39. Ahmad S, Callan R, Kossman R. Heparin reduction with citrate dialysate [abstract]. Nephrol Dial Transplant 2006; 21:suppl.4.

40. Chan AW, Tetzlaff JM, Altman DG, Dickersin K, Moher D. SPIRIT 2013: new guidance for content of clinical trial protocols. Lancet. 2013;381(9861):91-2.

41. Schulz KF, Altman DG, Moher D, CONSORT Group. CONSORT 2010 statement: updated guidelines for reporting parallel group randomised trials. Int J Surg. 2011:9(8):672-7.

\section{Submit your next manuscript to BioMed Central and take full advantage of:}

- Convenient online submission

- Thorough peer review

- No space constraints or color figure charges

- Immediate publication on acceptance

- Inclusion in PubMed, CAS, Scopus and Google Scholar

- Research which is freely available for redistribution 